\title{
LA APORÍA DEL SOBERANO ENCADENADO. ANÁLISIS CRÍTICO DE «LAS LIMITACIONES JURÍDICAS AL SOBERANO» DEL PROFESOR ERNESTO GARZÓN VALDÉS*
}

\author{
Alberto Carrió Sampedro \\ Universidad de Oviedo
}

RESUMEN. En el presente artículo se realiza un breve comentario de los trabajos que el profesor Ernesto Garzón Valdés ha dedicado a analizar los problemas que plantea la limitación jurídica del soberano legal. Un tema, por otra parte, de perenne actualidad debido a la restricción a la que se ve sometida la acción del legislador en los modernos Estados democráticos de Derecho. La principal conclusión a la que se llega aquí - que es, a su vez, la divergencia principal con respecto a lo expuesto por Garzón Valdés- es que el concepto de soberanía es ya en sí mismo un concepto normativo. Un concepto que exige, por tanto, tener en cuenta las dos dimensiones que se engloban dentro del mismo por lo que referirse a él únicamente en términos formales conduce inevitablemente a una aporía lógica.

Palabras clave: Ernesto Garzón Valdés, soberanía, restricciones legislativas.

ABSTRACT. This article makes a short remark on Professor Ernesto Garzón Valdés's works about the problem of legal limitation of sovereignty. This is obviously a permanent problem because of the legislative restrictions in the modern constitutional States. The main conclusion in this article which is, at the same time, the main divergent point regarding Garzón Valdés's opinion- is that the sovereignty concept is in itself a normative concept. A concept that claims for its two dimensions and about which is not possible to speak only in formal terms, because it leads necessary into a logical «aporia».

Keywords: Ernesto Garzón Valdés, sovereignty, legislative restrictions.

* Sirva este breve comentario como homenaje y cumplimiento del deber —no sé si positivo pero desde luego sí general — de agradecimiento, y como reconocimiento de la deuda que tantas generaciones de iusfilósofos hemos contraído con el profesor Ernesto GARZÓN VALDÉs, de quien tanto hemos aprendido y cuyas obras, a buen seguro, habrán de seguir formándonos. 


\section{LA SOBERANÍA ENCADENADA}

$\mathrm{E}$

1 problema de la soberanía, que bien podría ser calificado de fundacional para la filosofía política y jurídica, así como la posibilidad de su limitación legal mediante normas a las que quede sujeta la acción del soberano es, nada menos, con el que se enfrenta el profesor GARZÓN VALDÉS en los trabajos que aquí se tratará de comentar ${ }^{1}$.

Para ello parte el profesor GARZÓN VALDÉS de la definición clásica de soberanía realizada por AUSTiN ${ }^{2}$, según la cual, como es sabido, ésta queda definida por dos notas contrapuestas: i) la «positiva», que identifica al soberano con la persona o grupo cuya autoridad es obedecida por todos o la mayoría de la población y ii) la «negativa», que indica que éste no obedece a ninguna otra autoridad. Como consecuencia de ello, afirma GARZÓN VALDÉS, siguiendo a AUSTIN, cualquier tipo de limitación jurídica que se pretenda realizar de una autoridad soberana con estas características debería rezar del siguiente modo: i) cada vez que se produzca el caso de que el soberano ordene P no se le debe obedecer, ii) el soberano ordena P. Como es notorio, lo anterior conduce a una imposibilidad pragmática ya que la segunda orden contradice a la primera, por lo que ésta sería de cumplimiento imposible ya que quedaría revocada por la segunda. El ejemplo del que se sirve GARZÓN VALDÉS para exponerlo más didácticamente es el de Odiseo (Ulises) encadenado que solicita de sus marineros que lo liberen de sus ataduras toda vez que considera que ha pasado el peligro que había motivado la orden precedente. Es obvio que Ulises contradice con esta orden la primera dado que en ella se expresaba claramente que sus subordinados no deberían liberarlo del mástil hasta que se hubieran alejado lo suficiente de la Isla de las Sirenas ${ }^{3}$. ¿Qué deben hacer los marineros?

Todo ello no constituye en realidad sino una disculpa de la que, a modo de preámbulo, se sirve GARZÓN VALDÉS para introducir el problema real que le interesa dilucidar. Y tal problema no es otro que el de la limitación de la actividad del legislativo que se realiza en no pocos de los actuales Estados constitucionales de Derecho. El ejemplo positivo, real, al que remite GARZÓN VALDÉS, es el que se plantea en relación con la norma dictada por el poder constituyente de la República Federal de Alemania (art. 79.3) que califica de ilícita toda modificación de la Ley Fundamental que afecte a la Federación en Estados, a los fundamentos de la participación de los Länder en la potestad legislativa o a los principios establecidos en los arts. $1 .^{\circ}$ y 20 de la misma ${ }^{4}$.

${ }^{1}$ GARZÓN VALDÉS, 1981: «Acerca de las limitaciones legales al soberano legal», en Sistema. Revista de Ciencias Sociales, núms. 43-44, pp. 43-57; GARZÓN VALDÉS, 1983: «Acerca de las limitaciones jurídicas del soberano», en Bulygin, Farell, Nino y Rabossi (comps.), El lenguaje del Derecho. Homenaje a Genaro R. Carrió, Buenos Aires: Abeledo Perrot, pp. 157-180; GARZÓN VALDÉS, 1993: «Las limitaciones jurídicas del soberano», en Derecho, ética y política, Madrid: Centro de Estudios Constitucionales, pp. 181-200.

2 Austin, 1970: [1861], The Province of Jurisprudence Determined, New York: Burt Franklin (reimp.), pp. 254 ss.

${ }^{3}$ El problema es presentado también en términos de la relación de autoridad a que queda sometido el hijo con respecto al padre por Ross, 1969: [1967], «On Self- Reference and a Puzzle in Constitutional Law», en Mind, n. ${ }^{\circ} 78$, pp. 1-24, pp. 8 y ss.; hay trad. esp., a cargo de BulYGIN, y GARZÓN VALDÉs, 1993: «Sobre la autoreferencia y un difícil problema de Derecho constitucional», en El concepto de validez y otros ensayos, México D.F.: Fontamara, 2. ${ }^{a}$ ed., pp. 47-81.

${ }^{4} \mathrm{El}$ art. 1 se refiere a la dignidad del hombre, al reconocimiento de los derechos que le son inviolables y a la consiguiente vinculación de los tres Poderes del Estado a los Derechos Fundamentales. Por su parte, el art. 20 
En definitiva, nos encontramos aquí no sólo con una restricción del principio democrático, similar aunque sin duda más estricto que el que se produce en otros textos constitucionales — cuya finalidad, como advierte GARZÓN VALDÉS, sería la de garantizar la pervivencia del orden constitucional frente a despotismos similares a los ya experimentados en Alemania-, sino con lo que quizá resulta más sorprendente: un derecho de resistencia para el caso de que se produzca una violación de la prohibición de reforma del orden constitucional. En otros términos, se reconoce a todo ciudadano alemán, como sanción máxima frente a la tiranía legal o a la usurpación ilegítima del poder -i.e., los dos casos clásicos que la Escolástica reconocía como condiciones para el uso del derecho de resistencia-, su legítima capacidad para oponerse a ellos ${ }^{5}$. Lo paradójico del caso es que este derecho de resistencia, de formulación expresamente positiva, nunca podría ser alegado jurídicamente sino tan sólo ejercido en términos fácticos ya que, como muy bien advierte el propio GARZÓN VALDÉS, se encuentra más allá del orden pacífico estatal.

De todas formas, llegados a este punto, la cuestión central que nos ocupa, i.e., la de la posibilidad de limitar legalmente la acción del soberano, permanece aún sin resolver. Una posible vía para la solución del asunto se encontraría en la definición que ofrece VON WRIGHT de la autoridad suprema. Según ésta, puede considerarse soberano al agente cuyo acto normativo «no es contenido de ninguna otra norma superior» ${ }^{6}$. En otros términos, soberano sería aquél cuya competencia para dictar normas no venga reconocida por ninguna otra norma anterior. Tal agente debería pues ser considerado como el legislador originario del sistema y sus actos normativos no serían revocables ni susceptibles de juicios o tachas de invalidez ya que serían precisamente éstos los que permitirían referirse a la revocabilidad e invalidez de las normas subsiguientes. Este agente es al que GARZÓN VALDÉS denomina soberano 0 para distinguirlo, siguiendo a BRYCE, del soberano ${ }_{1}$ que se correspondería con aquel individuo o grupo al que tal poder soberano le otorga la capacidad para regular jurídicamente la vida en comunidad. Los actos normativos que dictara este último vendrían autorizados por las normas originarias del sistema y éstas sí podrían ser revocadas o ser objeto de tachas de invalidez con respecto a aquéllas. En definitiva, mientras que no tendría sentido preguntarse por las limitaciones jurídicas del soberano sí estaría justificado hacerlo en el caso del soberano ya que este último quedaría sujeto a las limitaciones jurídicas que le impusiera aquél para el desarrollo de su actividad legislati$\mathrm{va}^{7}$. El concepto de autoridad suprema (en ejercicio) quedaría de este modo vinculado con el de autorización. La distinción entre uno y otro tipo de soberano evitaría el problema de regreso al infinito así como una cierta garantía, si bien es cierto que nunca del todo

\footnotetext{
hace referencia al carácter federal, democrático y social del Estado (ap. 1. ${ }^{\circ}$ ); a la soberanía popular ejercida a través de los órganos representativos y del procedimiento electoral (ap. 2. ${ }^{\circ}$ ); a la vinculación del Poder Legislativo a la Constitución y al ejercicio del Ejecutivo y del Judicial conforme a la legislación vigente (ap. 3. ${ }^{\circ}$. Hay, además, un último apartado $\left(4{ }^{\circ}\right)$, añadido en junio de 1968 , que concede a cualquier nacional el derecho de resistencia, cuando no exista otro remedio, contra quienquiera que se proponga eliminar el orden constitucional.

${ }^{5}$ Lo que interpretado en clave de lo que ATIENZA y RUIZ MANERO denominan «reglas que confieren poderes» otorgaría a los destinatarios la curiosa posibilidad de realizar una acción de imposible reconocimiento jurídico; cfr. Atienza y Ruiz Manero, 2004: Las piezas del Derecho, Barcelona: Ariel, 2. ${ }^{a}$ ed., pp. 83 y ss.

${ }^{6}$ Cfr., von Wright, 1963: Norm and Action. A Logical Enquiry, London: Routlegde \& Kegan Paul, p. 192.

${ }^{7}$ Más recientemente PÉREZ TRIVIÑo ha descrito la relación que se produce entre poder originario y delegado en términos de lo que él denomina «autoridad suprema continua»; cfr. PÉREZ TrIVIÑo, 1998: Los límites jurídicos al soberano, Madrid: Tecnos, pp. 143 y ss.
} 
suficiente, contra el absolutismo. Dicho en otros términos, aunque el soberano ${ }_{1}$ pudiera sentirse tentado a violar las normas que le otorgan competencia serían estas mismas normas las que, para poder seguir gozando de su posición, le obligarían de algún modo a «tener que querer» el mantenimiento del orden constitucional establecido.

\section{UNA OBSERVACIÓN CRÍTICA}

El concepto de soberanía así como el de autoridad suprema son conceptos complejos que no pueden ser resueltos apelando únicamente a criterios lógicos debido a que la autorreferencialidad que ello conlleva conduce necesariamente a contradicciones o a un regreso al infinito ${ }^{8}$, como por otra parte había mostrado ya perfectamente AUSTIN siguiendo la tradición iniciada por BODIN y que pasando por HOBBES llega a KANT y se prolonga hasta Ross ${ }^{9}$. Para solucionarlo bien puede acudirse a la distinción entre soberano y soberano como hace GARZÓN VALDÉS, a la norma fundamental kelseniana o la norma básica de Ross. Pero con este tipo de estipulaciones, que quizá permitan encontrar una vía de escape a la paradoja lógica planteada con la posibilidad de limitar legalmente al soberano, no se consigue resolver el problema ya que de aceptarlas habría que admitir también que el soberano ${ }_{1}$ no es realmente un soberano - como no lo es el legislador que encuentra límites insalvables en la Constitución ni el poder de reforma de ésta que sigue las propias normas establecidas en ella para realizarla-. Dicho de otro modo, con ello tan sólo se consigue descomponer el problema en dos elementos uno estático y otro dinámico que no pueden sino operar conjuntamente. Algo similar es lo que ocurría con la tesis del Acto Puro del Primer Motor Inmóvil que, proveniente de ARISTÓTELES, fue defendida por la filosofía tomista ${ }^{10}$. Pero esta última, al igual que ocurre en el caso de la soberanía, no es una tesis «exenta», una proposición analítica que pueda demostrar su evidencia por sí misma (a priori) sino que, como muy bien afirmaba Tomás DE AQUINO — y en el caso de la soberanía todos los autores mencionados, incluido el propio GARZÓN VALDÉS-, es una tesis relativa a las cosas que se mueven, a las que, por decirlo en términos escolásticos, así se nos aparecen en nuestra «experiencia sensible» (ensu constat, como escribía Tomás DE AQUINO).

En resolución, el concepto de soberano o de autoridad suprema es ya en sí mismo un concepto normativo y como tal viene definido por dos dimensiones indesligables, a saber, la representativa y la ejercitativa a través de las cuales es posible dar cuenta del campo referido a las acciones humanas (prákta ${ }^{11}$. La primera es la que permite hablar

${ }^{8}$ No se analizará aquí la distinción, puesta ya de manifiesto por ARISTÓTeles (cfr. ARISTÓTELES, Política, trad. esp. a cargo de GarCía GuAl y PÉREZ JimÉNEZ, 2004: Madrid: Tecnos, L. II, cap. 6, 1265a, p. 169), entre soberanía interna y externa.

${ }^{9}$ Cfr. Austin, 1970: 254 y ss.; Bodin, 1961: [1583], Les six Livres de la Repúblique, Aalen: Scientia, p. 132; HobBes, 1991: [1651], Leviathan, TucK (ed.), Cambridge: Cambridge University Press, Cap. XVIII, \$90-91, pp. 123-5; KANT, 1968: [1793], «Über den Gemeinspruch: "Das mag in der Theorie richtig sein, taugt aber nicht für die Praxis"», en 1968: Kants Werke. Akademie Textausgabe, Berlin: Walter de Gruyter, vol. VIII, pp. 273-313, pp. 300 y ss.; Ross, 1969: 5-8 (ed. esp. pp. 53-7).

${ }^{10}$ En relación con la comparación efectuada entre Dios y el soberano terrenal $c f r$. DE AQUiNO, Santo Tomás, 1960: Summa Theologiae, Madrid: Biblioteca de Autores Cristianos, I, q. 2-3.

${ }^{11}$ Esas acciones que, como afirmaba ARISTÓTELES, dan lugar distintas formas de ser o de realización de una acción que puede ser de una u otra manera; $c f r$. ARISTÓTELES, 1999: Ética a Nicómaco, ARAUjo, y MARÍAS (eds.), Madrid: Centro de Estudios Constitucionales, 1140b, p. 92. 
de representaciones teoréticas del acto ejercitado en primer lugar — del acto de producir normas sin que éstas vengan autorizadas por ninguna otra norma anterior- aunque no por ello tiene que ser éste necesariamente entendido en términos de un acto prístino para todo momento y lugar sino originario de cada comunidad política. La segunda es la que se refiere a los actos ejercitativos posteriores en tanto que éstos pueden verse como una reproducción —nunca repetición - del acto originario, pero dados ya en un contexto totalmente diferente; en una estructura política y jurídica en la que quedan envueltos y que dará lugar a otros actos que sin perder la referencia con respecto al primero se abren a ejercicios normativos distintos que en ocasiones pueden llegar a ser más amplios y complejos que el primero. Pero, si esto es así, lo que verdaderamente determina la pervivencia de la autoridad reconocida como soberana en cada comunidad es una cuestión de hecho, una práctica social colectiva en la que lo importante será que esa autoridad suprema continúe siendo reconocida como tal por todos aquellos agentes que se encuentran implicados en la obediencia y aplicación de sus normas ${ }^{12}$.

En resolución, todo ello aplicado al caso que aquí nos ocupa conduce a reconocer en la forma «constitución» no sólo una fuente que desde el punto de vista de la teoría del Derecho se sitúa en el escalafón jerárquico superior sino lo que es aun más importante: que tal forma jurídica expresa el compromiso de que los proyectos políticos sean canalizados jurídicamente dentro de los márgenes —y respetando los valores-que ésta establece. Ahora bien, referirse a la Constitución en estos términos exige, antes que otra cosa, tener en cuenta la práctica social que la respeta y reconoce como orden de conducta vigente en cada sociedad de referencia ${ }^{13}$. En este sentido es en el cabe pues afirmar que su pervivencia nunca podrá ser asegurada por ella misma ya que dependerá en todo momento de esa práctica colectiva. Dependerá, en definitiva, de una cuestión de hecho y, siendo esto así, como acertadamente advirtiera HART, preguntarse en términos formales por el carácter de una norma que prescribe su propia obediencia no conduce — desde el punto de vista de la teoría del Derecho— más que a una tautología ${ }^{14}$.

${ }^{12}$ Cfr. HART, 1994: The Concept of Law, Oxford: Oxford University Press, 2. ${ }^{a}$ ed., pp. 152-154.

${ }_{13}$ No podemos detenernos aquí a analizar en extenso los problemas que ello puede plantear para el positivismo jurídico. En relación con estos y otros factores - políticos y jurídicos- que inciden en la pervivencia de las constituciones vid., FERRERES, 2000: «Una defensa de la rigidez constitucional», en Doxa, n. ${ }^{\circ} 23$, pp. 29 47, pp. 30 y ss; AGUILÓ, 2001: «Sobre la constitución del Estado constitucional», en Doxa, n. ${ }^{\circ} 24$, pp. 429-457, pp. 437 y ss. y BAYÓN, 2004: «Democracia y derechos: problemas de fundamentación del constitucionalismo», en Betegón, De PÁramo, Prieto Sanchos y Laporta (coords.), Constitución y Derechos Fundamentales, Madrid: Ministerio de la Presidencia/Centro de Estudios Políticos y Constitucionales, pp. 67-138, pp. 84 y ss.

${ }^{14}$ Cfr. HART, 1994: 109-110 y 293, notas 2 y 3. 